\title{
SYNTHESIS OF THE MULTIFERROIC La2CoMnO6 BY THE PROTEIC SOL-GEL METHOD
}

\author{
Miranda, M. A. R.*; Martins, A. F. N.; Dias, D. F.; Sasaki, J.M. \\ Departamento de Física, Universidade Federal do Ceará, Fortaleza, Ceará, Brazil \\ *marcus.a.r.miranda@gmail.com
}

\begin{abstract}
Multiferroics are materials that present two or three of the ferroic orders (ferroelectricity, (anti)(ferri)ferromagnetism and ferroeleasticity) simultaneously. These materials are interesting when there is a coupling between the ferroic orders, such as magnetic field driven polarization or electric field driven magnetization, which has several applications such as in information storage devices where one can devise a 4-bit memory state, or reduce power consumption by writing using an electric field and reading using a magnetic field. Possible multiferroics include thin film or policrystalline composites, in which each phase of the composite contributes with a ferroic order and single phase materials in which the sources of ferromagnetism and ferroelectricity coexists. Among these single phase materials are the complex oxides, such as double perovskites, in which one of the cations contributes with magnetism and the other with ferroelectricity. In this work we present preliminary $\mathrm{x}$-ray powder diffraction results in the synthesis of $\mathrm{La}_{2} \mathrm{CoMnO}_{6}$ (LCMO) by the proteic sol-gel method developed by our group. The LCMO crystal belongs to the double perovskites class of the type $\mathrm{A}_{2} \mathrm{~B}{ }^{\prime} \mathrm{B}^{\prime} \mathrm{O}_{6}$ ( $\mathrm{A}$ is usually a rare earth and $\mathrm{B}$ ' and $\mathrm{B}$ " are transition metals) and is a possible multiferroic. We managed to synthetize LCMO at temperatures of $600{ }^{\circ} \mathrm{C}$ and $700{ }^{\circ} \mathrm{C}$.
\end{abstract}

